\title{
Caracterización de una población pediátrica seguida en una clínica de anticoagulación
}

\author{
Description of a pediatric population followed at an anticoagulation clinic
}

\author{
Cesar H. Cely ${ }^{1,2}$, Stephania Galindo-Coral2,3,4, Carolina Mueses², Luis Figueroa², Liliana J. Flórez ${ }^{2,4}$ y \\ Juan E. Gómez-Mesa $2,3,4$ * \\ ${ }^{1}$ Servicio de Cardiología Pediátrica, Fundación Valle del Lili; ${ }^{2}$ Facultad de Medicina, Universidad Icesi; ${ }^{3}$ Servicio de Cardiología Adultos, Fundación \\ Valle del Lili; ${ }^{4}$ Centro de Investigaciones Clínicas, Fundación Valle de Lili. Cali, Colombia
}

\begin{abstract}
Resumen
Introducción: En la población pediátrica, el 95\% de las tromboembolias venosas son secundarias a un factor de riesgo; la presencia de catéter venoso central es el factor de riesgo más importante para el desarrollo de trombosis venosa profunda en esta población. Los estudios en población adulta sobre el uso de anticoagulantes se han extrapolado a la población pediátrica, y por ello es importante tener en cuenta las características diferenciales entre ambas poblaciones. Método: Se realizó un estudio descriptivo en el que se revisaron 2300 registros clínicos de pacientes seguidos por la Clínica de Anticoagulación institucional desde 2011 hasta 2019, buscando identificar pacientes menores de 18 años que hubieran recibido manejo anticoagulante ambulatorio. Resultados: Se encontraron 43 pacientes menores de 18 años, el 60\% de sexo femenino, con una edad promedio de $9.8 \pm 5.2$ años. El antecedente patológico más prevalente fueron las valvulopatías (28\%). Las principales indicaciones para recibir manejo anticoagulante incluyeron cardiopatías congénitas (21\%), prótesis valvular cardíaca mecánica (21\%, 5 aórticas y 4 mitrales) y trombosis venosa profunda (14\%). La warfarina se utilizó en el $72 \%$ de Ios pacientes. El $81 \%$ de los pacientes recibieron tratamiento anticoagulante extendido. Las principales complicaciones durante el tratamiento anticoagulante fueron sangrado ginecológico y cutáneo, así como trombocitopenia. Conclusiones: Las cardiopatías congénitas y las valvulopatías cardíacas son las principales indicaciones para considerar el manejo anticoagulante en la población pediátrica. La warfarina es el medicamento más utilizado en forma ambulatoria en esta población. La adherencia adecuada y el seguimiento ambulatorio en esta población requieren herramientas adicionales para lograr un excelente tratamiento anticoagulante.
\end{abstract}

Palabras clave: Anticoagulación. Clínica de anticoagulación. Pediatría. Warfarina. Enoxaparina.

\section{Abstract}

Introduction: In pediatric patients, 95\% of venous thromboembolisms are secondary to a risk factor; the presence of central venous catheter is the most important risk factor for the development of deep vein thrombosis in this population. Studies about anticoagulation treatment in adult patients have been extrapolated to the pediatric population, it is important to consider the differential characteristics between these two populations. Method: A descriptive study was conducted; there were reviewed 2300 clinical records of patients followed by the institutional anticoagulation clinic from 2011 to 2019, looking for underage patients who have received ambulatory anticoagulation treatment management. Results: 43 patients under the age of 18 years

\section{Correspondencia:}

*Juan E. Gómez-Mesa

E-mail: juan.gomez.me@fvl.org.co
Fecha de recepción: 19-08-2020

Fecha de aceptación: 07-10-2020

DOI: 10.24875/RCCAR.M22000119
Disponible en internet: 22-02-2022 Rev Colomb Cardiol. 2022;29(1):64-69 www.rccardiologia.com 0120-5633 / @ 2022 Sociedad Colombiana de Cardiología y Cirugía Cardiovascular. Publicado por Permanyer. Este es un artículo open access bajo la licencia CC BY-NC-ND (http://creativecommons.org/licenses/by-nc-nd/4.0/). 
old were found. $60 \%$ were female and the average age was $9.8 \pm 5.2$ years. The most prevalent pathological antecedent was valve disease (28\%). The main indications for anticoagulant treatment included congenital heart disease $(21 \%)$, mechanical heart valves (21\%, 5 aortic and 4 mitral) and deep vein thrombosis (14\%). Warfarin was found in $72 \%$ of patients. $81 \%$ of patients received extended anticoagulation therapy. The main complications during anticoagulant treatment included gynecological and cutaneous bleeding, as well as thrombocytopenia. Conclusions: Congenital heart disease and heart valve disease are the main indications for considering anticoagulation treatment in the pediatric population. Warfarin is the most formulated anticoagulant in this outpatient population. Adequate adherence and outpatient follow-up in requires additional tools to achieve excellent anticoagulant treatment.

Keywords: Anticoagulation. Anticoagulation clinic. Pediatrics. Warfarin. Enoxaparin.

\section{Introducción}

La incidencia de tromboembolia venosa (TEV) en pacientes pediátricos ha aumentado en los últimos 20 años debido a la aparición de tratamientos e intervenciones quirúrgicas que prolongan la vida de pacientes con enfermedades crónicas o por condiciones clínicas que hace algunas décadas eran consideradas incompatibles con la vida ${ }^{1,2}$.

A diferencia de los adultos, el 95\% de los episodios de TEV en niños son secundarios a un factor de riesgo identificable. El uso de catéter venoso central (CVC) se considera uno de los principales factores de riesgo para el desarrollo de trombosis venosa profunda (TVP) en niños ${ }^{3}$. Los casos de TEV, en aproximadamente el $90 \%$ de los recién nacidos y más del $50 \%$ de los niños están asociados a un $\mathrm{CVC}^{4}$. También se consideran otras condiciones para anticoagulación pediátrica, como cirugías de corrección de cardiopatías congénitas, cáncer y reemplazo valvular cardíaco'.

Se ha intentado extrapolar la evidencia aportada por diferentes estudios en población adulta sobre el uso de anticoagulantes a la población pediátrica ${ }^{5}$; sin embargo, es importante tener en cuenta las características diferenciales que existen entre estas dos poblaciones, entre ellas: 1) el sistema de coagulación se considera inmaduro en niños menores de 1 año; 2) la vía de administración del anticoagulante en pacientes de esta edad es compleja, ya sea oral o subcutánea; y 3) la polifarmacia en pacientes pediátricos críticamente enfermos o con múltiple comorbilidad incrementa el riesgo de sangrado y de trombosis debido a las interacciones medicamentosas $s^{6,7}$.

Para el tratamiento de la TEV en la población pediátrica, los anticoagulantes empleados son heparina no fraccionada, heparina de bajo peso molecular (HBPM) $\mathrm{y}$ antagonistas de la vitamina $\mathrm{K}$, principalmente warfarina $^{8}$. La HBPM es la preferida en entornos tanto hospitalarios como ambulatorios, debido a que su farmacocinética es menos variable en comparación con la heparina no fraccionada, por lo que requiere menos ajustes y una monitorización menos estricta; sin embargo, la aplicación inyectada diaria es una desventaja en los pacientes pediátricos ${ }^{1}$. Los antagonistas de la vitamina $\mathrm{K}$ tienen numerosas interacciones farmacológicas y un índice terapéutico muy estrecho que aumenta el riesgo de hemorragia grave ${ }^{9}$, pero su vía de administración oral es una ventaja en la población pediátrica.

A pesar de que existen pautas de tratamiento recomendadas para el manejo de la anticoagulación pediátrica ${ }^{10}$, aún no hay suficiente evidencia e información para la creación de un protocolo específico de manejo en esta población ${ }^{11}$. De igual forma, dadas las limitaciones acerca del uso de los anticoagulantes clásicos (antagonistas de la vitamina $\mathrm{K}$ y heparinas) en pediatría, hay un gran interés por los anticoagulantes orales directos; no obstante, los estudios clínicos con estos medicamentos en población pediátrica aún deben esclarecer si tienen un balance positivo respecto a eficacia y seguridad en los diferentes grupos de edad ${ }^{8}$.

Teniendo en cuenta que la Fundación Valle del Lili es un centro de referencia de servicios de alta complejidad en Colombia y Latinoamérica, existe la necesidad de documentar y recopilar datos sobre la experiencia con pacientes pediátricos de la Clínica de Anticoagulación. Este estudio sirve como punto de partida para la realización de investigaciones futuras, que permitan determinar la dosificación óptima de un anticoagulante y establecer o identificar posibles interacciones farmacológicas, efectos adversos, complicaciones, desenlaces y seguimiento, entre otros.

\section{Método}

Se llevó a cabo un estudio de tipo descriptivo en el cual se incluyeron 43 pacientes menores de 18 años seguidos por la Clínica de Anticoagulación de la Fundación Valle del Lili atendidos desde el 1 de enero de 2011 hasta julio de 2019. El estudio fue aprobado por 
el Comité de Ética e Investigación Biomédica de la Fundación Valle del Lili. Teniendo en cuenta que no se realizaron intervenciones adicionales con propósito del estudio y únicamente se hizo recolección de la información de cada paciente, este estudio no requirió el diligenciamiento de consentimiento informado.

Se revisó la historia clínica de cada paciente inscrito en el servicio de Clínica de Anticoagulación de la institución en busca de los datos requeridos para este estudio; los datos fueron ingresados a una base de datos personalizada, la cual es administrada y protegida por el Centro de Investigaciones Clínicas de la Fundación Valle del Lili.

Entre las limitaciones observadas cabe resaltar que la base de datos utilizada cuenta con varios digitadores debido a que se corren diferentes estudios a partir de la misma base de datos, lo cual puede presentar un sesgo para los resultados. Sin embargo, se realizaron controles durante la recolección de datos para asegurar, en lo posible, una buena calidad de los mismos.

Para el procesamiento y el análisis de los datos se utilizaron las herramientas de la estadística descriptiva; las variables categóricas se presentaron en frecuencias y porcentajes. Para el análisis de datos se utilizó el programa Stata 12.1.

\section{Resultados}

Se registró información de 43 pacientes pediátricos, de los cuales $26(60 \%)$ corresponden a mujeres, $40(93 \%)$ son de raza mestiza y tan solo $2(5 \%)$ provienen de zonas rurales (Tabla 1). Entre los antecedentes patológicos más prevalentes se incluyen 12 valvulopatías cardíacas $(28 \%)$, siendo la más común la estenosis aórtica grave (4 pacientes); la cirugía valvular aórtica se presentó en el $14 \%$ y la cirugía valvular mitral en 5 pacientes (11\%). Otros antecedentes patológicos fueron estado hipercoagulable en 7 pacientes (16\%), hipertensión arterial en 7 pacientes (16\%), insuficiencia cardíaca en 6 pacientes (14\%) y cáncer en 4 pacientes (9\%) (Tabla 2).

La indicación para anticoagulación incluyó principalmente cardiopatías congénitas en 9 casos (21\%), prótesis valvulares mecánicas (21\%; aórtica en 5 pacientes y mitral en 4 pacientes) y TVP en 6 casos (14\%) (Tabla 3). En 3 pacientes con antecedente de TVP se identificaron factores de riesgo asociado a TVP, los cuales incluyen hospitalización en los 3 meses previos al evento de TEV en 1 paciente (2\%), cirugía en los 3 meses previos en 1 paciente (2\%) y uso de CVC en la ubicación anatómica de la TVP (braquial) en 1 paciente (2\%) (Tabla 4).
Tabla 1. Caracterización sociodemográfica

\begin{tabular}{|c|c|}
\hline Características sociodemográficas & $\mathbf{n}=\mathbf{4 3}$ \\
\hline Edad, años* & $9.8 \pm 5.2$ \\
\hline $\begin{array}{l}\text { Sexo, } n \text { (\%) } \\
\text { Femenino } \\
\text { Masculino }\end{array}$ & $26(60)$ \\
\hline Raza, $n$ (\%) & $17(40)$ \\
Mestizo & \\
\hline Blanco & $40(93)$ \\
Afrodescendiente & $2(5)$ \\
Procedencia, $n(\%)$ & $1(2)$ \\
Urbano & \\
Rural & $41(95)$ \\
\hline
\end{tabular}

*Promedio \pm desviación estándar.

El principal tratamiento anticoagulante formulado fue warfarina, en 31 pacientes (72\%), seguido por HBPM (enoxaparina) en 12 pacientes (28\%). La duración del tratamiento anticoagulante se consideró como indefinido o extendido en 36 pacientes (84\%), y la adherencia se logró en el $98 \%$ de los pacientes. Solo 5 pacientes (11\%) habían recibido previamente otro medicamento anticoagulante, el cual se modificó por diferentes razones (Tabla 5).

Las complicaciones relacionadas con el tratamiento anticoagulante incluyeron hemorragia ginecológica, hemorragia cutánea, requerimiento de transfusión debido a hemorragia, requerimiento de hospitalización y trombocitopenia, en un paciente respectivamente $(2 \%$ para cada complicación) (Tabla 6).

\section{Discusión}

La literatura que aporta información respecto a la anticoagulación pediátrica es limitada. No obstante, se mencionan cuatro grupos de pacientes en los que se tiene una experiencia en este campo: 1) neonatos críticamente enfermos; 2) niños con cáncer (que requieren líneas centrales permanentes); 3) niños con anormalidades genéticas 0 adquiridas de la coagulación (proteína C, proteína S, deficiencia de antitrombina III, lupus anticoagulante y anticardiolipina); y 4) niños con ciertas formas de cardiopatía congénita y adquirida $^{12-14}$.

Los resultados obtenidos en este estudio permiten conocer el entorno del manejo anticoagulante en la población pediátrica en una institución de referencia nacional. La edad promedio de los pacientes fue de $9.8 \pm 5.2$ años y el sexo femenino fue el más 
Tabla 2. Antecedentes patológicos

\begin{tabular}{|c|c|}
\hline Antecedentes & $n=43$ \\
\hline Estado hipercoagulable/trombofilia, $n$ (\%) & $7(16)$ \\
\hline Enfermedad renal crónica, $n(\%)$ & $2(5)$ \\
\hline Insuficiencia cardíaca crónica, n (\%) & $6(14)$ \\
\hline FEVI disminuida & 4 \\
\hline FEVI preservada & 2 \\
\hline Valvulopatías, n (\%) & $12(28)$ \\
\hline Insuficiencia aórtica grave & 3 \\
\hline Estenosis aórtica grave & 4 \\
\hline Insuficiencia mitral grave & 3 \\
\hline Estenosis mitral grave & 2 \\
\hline Cirugía valvular aórtica, $n$ (\%) & $6(14)$ \\
\hline Plastia & 4 \\
\hline Prótesis mecánica & 2 \\
\hline Cirugía valvular mitral, n (\%) & $5(11)$ \\
\hline Prótesis mecánica & 4 \\
\hline Prótesis biológica & 1 \\
\hline Otra valvulopatía grave, $n$ (\%) & $4(9)$ \\
\hline Tricúspide & 1 \\
\hline Pulmonar & 3 \\
\hline Hipertensión arterial sistémica, n (\%) & $7(16)$ \\
\hline Diabetes mellitus, $n$ (\%) & $2(5)$ \\
\hline Enfermedad coronaria, $n$ (\%) & $3(7)$ \\
\hline Cáncer, n (\%) & $4(9)$ \\
\hline Accidente cerebrovascular isquémico, $n(\%)$ & $3(7)$ \\
\hline
\end{tabular}

FEVl: fracción de eyección del ventrículo izquierdo.

prevalente, lo cual difiere de lo publicado en otros estudios, en los que se describe un pico bimodal en la incidencia de TEV, incluyendo el período neonatal en uno de ellos, y es más frecuente en población masculina $^{12-14}$.

En este estudio se encontró que entre las indicaciones de anticoagulación más frecuentes están las cardiopatías congénitas $(21 \%)$ y ser portador de prótesis valvular mecánica (21\%), lo cual concuerda con publicaciones en las que las indicaciones más reportadas fueron el reemplazo de válvula cardíaca (hasta el $23.6 \%$ ) y el procedimiento de Fontan (hasta el 19.5\%) ${ }^{9,15}$. No obstante, otro estudio prospectivo en pacientes con
Tabla 3. Indicaciones médicas para el inicio de anticoagulación

\begin{tabular}{|l|c|}
\hline Indicaciones & $n=43$ \\
\hline Fibrilación auricular (\%) & $1(2)$ \\
\hline No valvular & 1 \\
\hline Prótesis valvular mecánica (\%) & $9(21)$ \\
\hline Aórtica & 5 \\
\hline Mitral & 4 \\
\hline Prótesis valvular biológica (\%) & $2(4)$ \\
\hline Aórtica & 2 \\
\hline Trombosis venosa profunda (\%) & $6(14)$ \\
\hline Tromboembolia pulmonar (\%) & $3(7)$ \\
\hline Hipertensión pulmonar, $n(\%)$ & $3(7)$ \\
\hline Enfermedad cerebrovascular, $n$ (\%) & $3(7)$ \\
\hline Trombosis venosa cerebral, $n(\%)$ & $3(7)$ \\
\hline Foramen oval permeable, $n(\%)$ & $3(7)$ \\
\hline Cardiopatía congénita, $n(\%)$ & $9(21)$ \\
\hline
\end{tabular}

Tabla 4. Factores de riesgo asociados a tromboembolia venosa

\begin{tabular}{|l|l|}
\hline Factores de riesgo asociados a TEV & $\mathrm{n}=\mathbf{4 3}$ \\
\hline Otra cirugía en los 3 meses previos a TEV, $\mathrm{n}(\%)$ & $1(2)$ \\
\hline Hospitalización en los 3 meses previos a TEV, $\mathrm{n}(\%)$ & $1(2)$ \\
\hline Catéter venoso central en el lugar de TEV, $\mathrm{n}(\%)$ & $1(2)$ \\
\hline
\end{tabular}

TEV: tromboembolia venosa.

una media de edad de 36 meses mostró como principal indicación la TVP asociada a CVC e infección ${ }^{13}$.

La incidencia de TVP en niños es de 5 casos por 10,000 niños, pero se considera que es subdiagnosticada; su recurrencia se ha reportado entre el $6.5 \%$ y el $21 \%$ en niños con TEV. En este estudio, el $14 \%$ de los pacientes tenían diagnóstico de TVP. Adicionalmente, en la literatura se describe que el $95 \%$ de las TEV en población pediátrica son secundarias a un factor de riesgo identificable, principalmente el uso de $\mathrm{CVC}^{16}$. Un estudio retrospectivo de 346 pacientes menores de 1 mes con TEV mostró que el $65.6 \%$ tenían TVP relacionada con el $\mathrm{CVC}^{12}$. Por otro lado, en los registros de población adolescente (12-20 años) que desarrollaron TEV se observó como principal factor de riesgo la 
Tabla 5. Tratamiento anticoagulante instaurado

\begin{tabular}{|c|c|}
\hline Tratamiento & $n=43$ \\
\hline $\begin{array}{l}\text { Medicamento anticoagulante, } n(\%) \\
\text { Warfarina } \\
\text { Enoxaparina }\end{array}$ & $\begin{array}{l}31(72) \\
12(28)\end{array}$ \\
\hline $\begin{array}{l}\text { Cumplimiento de prescripción, } n(\%) \\
\text { No } \\
\text { Sí }\end{array}$ & $\begin{array}{c}1(2) \\
42(98)\end{array}$ \\
\hline $\begin{array}{l}\text { Manejo previo con otro anticoagulante, } n(\%) \\
\text { No } \\
\text { Enoxaparina } \\
\text { Warfarina }\end{array}$ & $\begin{aligned} 38 & (88) \\
4 & (9) \\
1 & (2)\end{aligned}$ \\
\hline $\begin{array}{l}\text { Razón de cambio anticoagulante, } n \text { (\%) } \\
\text { Decisión médica } \\
\text { Otra }\end{array}$ & $\begin{array}{l}3(7) \\
2(5)\end{array}$ \\
\hline $\begin{array}{l}\text { Duración sugerida anticoagulación, } n \text { (\%) } \\
3 \text { meses } \\
6 \text { meses } \\
\text { Extendida } \\
\text { Sin datos }\end{array}$ & $\begin{aligned} 5 & (12) \\
1 & (2) \\
36 & (84) \\
1 & (2)\end{aligned}$ \\
\hline
\end{tabular}

Tabla 6. Complicaciones desarrolladas durante el tratamiento anticoagulante

\begin{tabular}{|l|c|}
\hline Complicaciones durante la anticoagulación & $\mathrm{n}=\mathbf{4 3}$ \\
\hline Complicación hemorrágica ginecológica, $\mathrm{n}(\%)$ & $1(2)$ \\
\hline Complicación hemorrágica cutánea, $\mathrm{n}(\%)$ & $1(2)$ \\
\hline Complicación hemorrágica con hospitalización, $\mathrm{n}(\%)$ & $1(2)$ \\
\hline Complicación hemorrágica con transfusión, $\mathrm{n}(\%)$ & $1(2)$ \\
\hline Trombocitopenia, $\mathrm{n}(\%)$ & $1(2)$ \\
\hline Hospitalización, $\mathrm{n}(\%)$ & $1(2)$ \\
\hline
\end{tabular}

obesidad, en el $47 \%$ de los casos $^{17}$; sin embargo, tenían dos o más factores de riesgo al momento del diagnóstico. La aparición de TVP posterior a la colocación del CVC se observó en el $2 \%$ de la población, por lo cual se considera un factor de riesgo poco frecuente.

La incidencia de tromboembolia pulmonar (TEP) en niños es más baja que en adultos; no obstante, en cohortes de infantes y niños con enfermedad cardíaca congénita o adquirida existe mayor riesgo de desarrollar esta enfermedad como resultado de una homeostasis alterada, entre otros factores de riesgo generales ${ }^{18}$. En nuestro estudio, la TEP estaba presente en el 7\% de la población como indicación de anticoagulación. Cabe resaltar que en, nuestro caso, no es posible determinar si este evento es atribuible únicamente a la enfermedad cardíaca congénita ${ }^{18,19}$.
Entre los medicamentos anticoagulantes más usados en la población pediátrica están la heparina no fraccionada, la HBPM y la warfarina. Las heparinas generalmente se usan para un tratamiento corto o para el inicio del manejo a largo plazo con warfarina ${ }^{20}$; pese a ello, las HBPM han ganado popularidad por su efectividad demostrada, similar a la de la warfarina y la heparina no fraccionada, por su baja tasa de efectos adversos, incluyendo trombocitopenia y osteoporosis, y especialmente cuando se requieren períodos de anticoagulación cortos (3 meses) para el manejo de la $\mathrm{TEV}^{21}$. La warfarina continúa siendo otro de los medicamentos anticoagulantes más utilizados en la población pediátrica, aun cuando su manejo y titulación de dosis son particularmente difíciles en niños muy pequeños, en aquellos que reciben múltiples medicamentos y en quienes requieren intervenciones quirúrgicas frecuentes o con un alto riesgo de sangrado ${ }^{21-25}$. En nuestra población, el medicamento anticoagulante prescrito con más frecuencia fue la warfarina (72\%), seguido de la enoxaparina (28\%), lo cual puede estar influenciado por el tipo de pacientes manejados en el servicio, como son aquellos con cardiopatías congénitas que requieren terapia anticoagulante extendida.

El fondaparinux tiene ventajas en la población pediátrica, ya que permite una dosificación única diaria, no interfiere en el metabolismo óseo y no tiene riesgo de trombocitopenia inducida por heparina ${ }^{26}$. Existen varios reportes de casos y series respecto al uso de inhibidores directos de la trombina en esta población; sin embargo, la evidencia sobre efectos adversos es más pobre en comparación con los otros grupos de anticoagulantes ${ }^{27}$, por lo que su uso está limitado a casos de trombocitopenia inducida por heparina y con mucha precaución ${ }^{18}$.

En la literatura, el porcentaje de eventos hemorrágicos durante el tratamiento anticoagulante es variable y oscila entre el $4 \%$ y el 10\% 9,15 . En el caso de las HBPM, el sangrado mayor y el menor se han descrito hasta en el $2.9 \%$ y en el $23.4 \% 28$. En nuestro estudio se observaron complicaciones hemorrágicas en el $8 \%$ de los casos, entre las cuales se describen sangrado de origen ginecológico y cutáneo, además de hemorragia con necesidad de transfusión y hemorragia con requerimiento de manejo hospitalario.

\section{Conclusiones}

Las cardiopatías congénitas y las valvulopatías cardíacas (prótesis valvular mecánica) son las principales indicaciones para considerar el manejo anticoagulante en la población pediátrica. La warfarina es el principal 
medicamento utilizado en forma ambulatoria en esta población, seguido por la HBPM (enoxaparina). Si bien la presencia de complicaciones relacionadas con la anticoagulación, incluyendo complicaciones hemorrágicas, no contraindica la terapia anticoagulante, hace necesario implementar medidas adicionales para lograr una mayor adherencia y un seguimiento ambulatorio teniendo en cuenta las dificultades que pudieran existir en la implementación de este tratamiento en la población pediátrica (edad, sociales, culturales, entre otras).

\section{Financiamiento}

Los autores no recibieron financiamiento para este artículo.

\section{Conflicto de intereses}

Los autores no reportan conflictos de intereses.

\section{Responsabilidades éticas}

Protección de personas y animales. Los autores declaran que para esta investigación no se han realizado experimentos en seres humanos ni en animales.

Confidencialidad de los datos. Los autores declaran que han seguido los protocolos de su centro de trabajo sobre la publicación de datos de pacientes.

Derecho a la privacidad y consentimiento informado. Los autores han obtenido la aprobación del Comité de Ética para el análisis y publicación de datos clínicos obtenidos de forma rutinaria. El consentimiento informado de los pacientes no fue requerido por tratarse de un estudio observacional retrospectivo.

\section{Bibliografía}

1. Young G. Anticoagulation therapies in children. Pediatr Clin N Am. 2017;64:1257-69.

2. Raffini L, Huang Y, Witmer C, Feudtner $C$. Dramatic increase in venous thromboembolism in children's hospitals in the United States from 20012007. Pediatrics. 2009;124:1001-8.

3. Law C, Raffini L. A guide to the use of anticoagulant drugs in children. Paediatr Drugs. 2015;17:105-14.
4. Massicotte MP, Dix D, Monagle $P$, Adams M, Andrew M. Central venous catheter related thrombosis in children: analysis of the Canadian registry of venous thromboembolic complications. J Pediatrics. 1998;133:770-6.

5. Spentzouris G, Scriven RJ, Lee TK, Labropoulos N. Pediatric venous thromboembolism in relation to adults. J Vasc Surg. 2012;55:1785-93.

6. Andrew M, Vegh P, Johnston M, Bowker J, Ofosu F, Mitchell L. Maturation of the hemostatic system during childhood. Blood. 1992;80:1998-2005.

7. Ignjatovic V, Furmedge J, Newall F, Chan A, Berry L, Fong C, et al. Age-related differences in heparin response. Thromb Res. 2006;118:741-5.

8. Newall F, Branchford B, Male C. Anticoagulant prophylaxis and therapy in children: current challenges and emerging issues. J Thromb Haemost. 2018;16:196-208.

9. Moffett BS, Kim S, Bomgaars LR. Readmissions for warfarin-related bleeding in pediatric patients after hospital discharge. Pediatr Blood Cancer. 2013;60:1503-6.

10. Stem J, Christensen A, Davis D, Raffini L. Safety of prophylactic anticoagulation at a pediatric hospital. J Pediatr Hematol Oncol. 2013;35:e287-91.

11. Monagle P, Chan AKC, Goldenberg NA, Ichord RN, Journeycake JM, Nowak-Göttl U, et al. Antithrombotic therapy in neonates and children: antithrombotic therapy and prevention of thrombosis. $9^{\text {th }}$ ed. American College of Chest Physicians Evidence-Based Clinical Practice Guidelines. Chest. 2012;141(2 Suppl):e737S-e801S.

12. Chan A, Lensing AWA, Kubitza D, Brown G, Elorza D, Ybarra M, et al. Clinical presentation and therapeutic management of venous thrombosis in young children: a retrospective analysis. Thromb J. 2018;16:29.

13. Gupta T, Delhi Kumar CG, Ramesh Kumar R, Adhisivam B. Pediatric thrombotic events: incidence, clinical risk factors and outcome. Indian $\mathrm{J}$ Pediatr. 2019;86:241-4.

14. Reller MD. Congenital heart disease: current indications for antithrombotic therapy in pediatric patients. Curr Cardiol Rep. 2001;3:90-5.

15. Monagle $P$. Anticoagulation in the young. Heart. 2004;90:808-12.

16. Chan AK, Deveber G, Monagle P, Brooker LA, Massicotte PM. Venous thrombosis in children. J Thromb Haemost. 2003;1:1443-55.

17. Ishola T, Kirk SE, Guffey D, Voigt K, Shah MD, Srivaths L. Risk factors and co-morbidities in adolescent thromboembolism are different than those in younger children. Thromb Res. 2016;141:178-82.

18. Giglia M, Massicotte MP, Tweddell JS, Barst RJ, Bauman M, Erickson CC, et al. Prevention and treatment of thrombosis in pediatric and congenital heart disease a scientific statement from the American Heart Association. Circulation. 2013;128:2622-703.

19. Goldenberg NA, Durham JD, Knapp-Clevenger R, Manco-Johnson MJ. A thrombolytic regimen for high-risk deep venous thrombosis may substantially reduce the risk of postthrombotic syndrome in children. Blood. 2007;110:45-53.

20. Dabbous MK, Sakr FR, Malaeb DN. Anticoagulant therapy in pediatrics. J Basic Clin Pharm. 2014;5:27-33.

21. Chalmers E, Ganesen V, Liesner R, Maroo S, Nokes T, Saunders D, et al. Guideline on the investigation, management and prevention of venous thrombosis in children. Br J Haematol. 2011;154:196-207.

22. Monagle $\mathrm{P}$, Newall F. Anticoagulation in children. Thromb Res. 2012;130:142-6.

23. Chan AKC, Monagle P. Updates in thrombosis in pediatrics: where are we after 20 years? Hematol Am Soc Hematol Educ Progr. 2012;2012:439-43.

24. Bohnhoff JC, DiSilvio SA, Aneja RK, Shenk JR, Domnina YA, Brozanski BS, et al. Treatment and follow-up of venous thrombosis in the neonatal intensive care unit: a retrospective study. J Perinatol. 2017;37:306-10.

25. Goldenberg NA, Takemoto CM, Yee DL, Kittelson JM, Massicotte MP. Improving evidence on anticoagulant therapies for venous thromboembolism in children: key challenges and opportunities. Blood. 2015;126:2541-7.

26. Young $\mathrm{G}$, Male $\mathrm{C}$, van Ommen $\mathrm{CH}$. Anticoagulation in children: making the most of little patients and little evidence. Blood Cells Mol Dis. 2017;67:48-53.

27. Cetta F, Graham LC, Wrona LL, Arruda MJ, Walenga JM. Argatroban use during pediatric interventional cardiac catheterization. Catheter Cardiovasc Interv. 2004;61:147-9.

28. Nowak-Göttl U, Bidlingmaier C, Krümpel A, Göttl L, Kenet G. Pharmacokinetics, efficacy, and safety of LMWHs in venous thrombosis and stroke in neonates, infants and children. Br J Pharmacol. 2008;153:1120-7. 